\title{
Synergism of the action of some stabilisers against the destruction of polymer materials
}

\author{
Irina Zhukova ${ }^{1}$, Evgeniya Flik $^{1 * *}$, Elena Shubina ${ }^{1}$, Vladimir Mishurov ${ }^{1}$, and Ivan \\ Kashparov $^{1,2}$ \\ ${ }^{1}$ Don State Technical University, Gagarin Square, 1, Rostov-on-Don, 344000, Russia \\ ${ }^{2}$ Platov South-Russian State Polytechnic University (NPI), 132, str. Prosveshcheniya, 346428, \\ Novocherkassk, Russia
}

\begin{abstract}
The process of thermal-oxidative degradation of polymer materials has been studied on the example of polyethylene in the presence of quinones and / or nitroxyl radicals. The synergistic effect of the positive action of a mixture of stabilisers from a number of quinones and nitroxyl radicals on the destructive process of polyethylene has been established. It has been shown that the rate of degradation has significantly slowed down when an equimolecular mixture of 4-acetylamino-2,2,6,6tetramethylhexahydropyridine-1-oxyl and 2,6-di-tert-amyl-1,4cyclohexadienone inhibitors has been introduced into the polymer. The kinetics of the initiated thermo-destructive process of polyethylene oxidation in the presence and absence of stabilisers has been studied. A mechanism of joint action of stabilisers based on their interaction with radicals and the breakage of radical chains, as well as a mechanism for the regeneration of stabilisers, has been proposed.
\end{abstract}

\section{Introduction}

The production of polymer materials (PM) and products made from them is a costeffective and dynamically developing industry. The share of PM application in various industries and agriculture is steadily growing. The prevalence of the use of PM is related to their resistance to climatic factors, which determines their service life and possible areas of use [1-5].

During an operation of polymer products or parts, qualitative changes occur in them, which gradually lead to their ageing and to further destruction of the material. Destructive processes in PM are influenced by various physical impacts (load, light, heat, etc.), as well as chemical ones in the form of external aggressive reagents (ozone, oxygen, halogens, etc.) $[5,6]$. Under the influence of these factors, processes related to thermo-oxidative degradation occur in the PM. To slow down negative changes inside the material, a special substances-stabilisers can be introduced into the PM. Stabilisers (or inhibitors, InH), due to their specific behavior, can significantly reduce the rate of destruction and prevent the destruction of PM. The stabilisers (or inhibitors, $\mathrm{InH}$ ) introduced into the PM slow down the cross linking and / or breaking of the chain of macromolecules, slow down and / or

\footnotetext{
* Corresponding author: eaff@yandex.ru
} 
prevent oxidative processes, reduce discolouration, and improve the quality of finished plastic products [5-7]. The purpose of the stabilisers depends on the purpose of their protective action. They can be used as light stabilisers, work as antioxidants, or act as antiozanates $[4,5,8]$.

According to the mechanism of the stabilising action of antioxidants, chain and nonchain inhibition are distinguished. In the first case, the active centers of the process are deactivated, in the second -substances that lead to the destruction of PM [9, 10]. In the case of a chain (or radical) mechanism of thermo-oxidative destruction, the PM can be stabilised at temperatures of no more than $300^{\circ} \mathrm{C}$. The breakage of the growing oxidation chains occurs due to the interaction of the stabiliser (otherwise $\mathrm{InH}$ ) with radical particles of the type $\mathrm{RO} \bullet 2$ and $\mathrm{R} \bullet: \mathrm{RO} \bullet 2+\mathrm{InH}=\mathrm{ROOH}+\mathrm{In} \bullet$, with the formation of low-activity particles of the stabiliser In ${ }^{\circ}$. But an increase in temperature contributes to the activity of In $\bullet$ radicals, which leads to the subsequent initiation of processes of deeper destruction $[10,11]$.

To prevent it, a highly active stabilisers are used. Active stabilisers of thermodestructive oxidation processes in PM include substances with a mobile hydrogen atom. These are spatially hindered phenols (PFPS), well-known and widely used in industry ionol (2,6-di-tert-butyl-4-methylphenol), neozone-D (N-phenyl-2-naphthylamine) and other similar aromatic structures with various substituents in the ring.

Nitroxyl radicals (HP) show a high stabilising activity as they react rapidly with alkyl radicals of the $\mathrm{R} \cdot$ type [12]. With a lack of oxygen, the radicals of the 2,2,6,6-tetramethylhexahydropyridine (2,2,6,6-TMP) series are used to prevent the destruction of PM.

In practice, HP precursors are widely used - spatially hindered amines (PPA or HALS stabilisers) of the 2,2,6,6-TMP series, which generate HP under conditions of thermal and photo-oxidative destruction of PM.

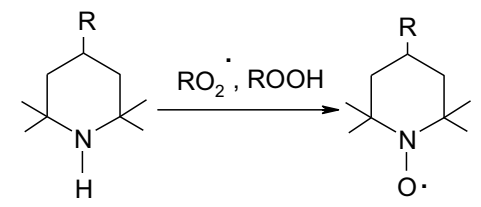

Fig. 1. Scheme.

HP, due to regeneration, can repeatedly break the chain and slow down the chain radical process:

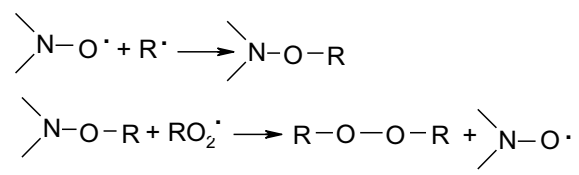

Fig. 2. Scheme.

Regeneration makes it possible to increase the efficiency of stabilisers by using them as low-concentration additives [2].

In order to protect the PM from thermo-destructive oxidation, mixed compositions of stabilisers are used. And in this case, the synergism of the action of these additives is of particular importance in the stabilisation of PM. The peculiarities of this phenomenon consist in the excess of the protective effect of the composition of stabilisers in comparison with the stabilising effect of individual components. A quantitative indicator of this effect is the bigger (longer) induction period of the InH mixture in comparison with the induction period of the individual most effective stabiliser. It should also be understood that an 
effective synergistic effect should be accompanied by permanent regeneration of the additives introduced for stabilisation $[4,6]$.

When creating a stabilising mixture composition with a targeted action, it should include substances-components that can destroy hydroperoxide compounds and do not form radical particles, as well as compounds that inhibit and/or break off chain radical reactions. Examples of such inhibitory systems and schemes of their operation are presented in a number of review papers $[2,4,6,9]$. The most active and technically used are recognized stabilising mixtures based on PFPS, sulphides, some amines and phosphites [4, 13]. It is promisingly to develop and study the mechanism of action of inexpensive mixed stabilisers in order to inhibit the processes of thermo-oxidative destruction in PM.

It should be noted that there is no data on the synergistic effect of a mixture of compounds from the class of PFP (or their derivatives) and HP on the stabilisation of PM in the literature. The most studied and most widely used are highly active stabilisers based on PFP - compounds with mobile hydrogen atoms, as well as HP, which can repeatedly break chains due to their regeneration.

It is of interest to study the synergistic effect of a mixture of stabilisers based on the oxidation products of PFP and HP, when one component will destroy hydro-peroxide compounds without the formation of radicals, and the other will break off radical chains and regenerate at the same time.

\section{Materials and methods}

Aldrich reagents were used without additional preparation; polyethylene (PE) of the HDPE 15803-020 brand (Russian Standard 16.337-77); 4-Acetylamino-2,2,6,6-tetramethylhexahydropyridine-1-oxyl (4-AcNH-TEMPO) was obtained according to the well-known method [14].

The model reaction was the oxidation of $\mathrm{PE}$ in the presence of azo-bis-isobutyronitrile (AIBN) when boiling with a reverse refrigerator in xylene $(\mathrm{T}=413 \mathrm{~K})$. The preparation of PE solutions and the determination of viscosity were carried out according to the method (Russian Standard 18249-72), as well as using a Brookfield DV1M rotary viscometer. A freshly prepared solutions were used. Extrapolation of the results of viscometry to the zero concentration of the polymer helped to determine the characteristic viscosity. A control solution (without a stabiliser) and solutions with the addition of a stabiliser were studied for the kinetics of thermal-oxidative degradation of $\mathrm{PE}$. The concentration of the initiator in the PE solution was $2 \cdot 10^{-3} \mathrm{~mol} / 1$, the concentration of the stabilisers was $3 \cdot 10^{-3} \mathrm{~mol} / 1$.

The stabilisers used were HP-4-amino-TEMPO (1), 4-AcNH-TEMPO (2), and quinones-2,6-di-tert-amyl-1,4-cyclohexadienone (3), 2,6-di-tert-butyl-1,4cyclohexadienone (4), 3,3',5,5'-tetra-tert-butyl-diphenoquinone (5) 3,3',5,5' -tetra-tert-butylstilbenquinone (6), as well as a mixture of $\mathrm{HP}$ and quinone in a ratio of $1 / 1$. 

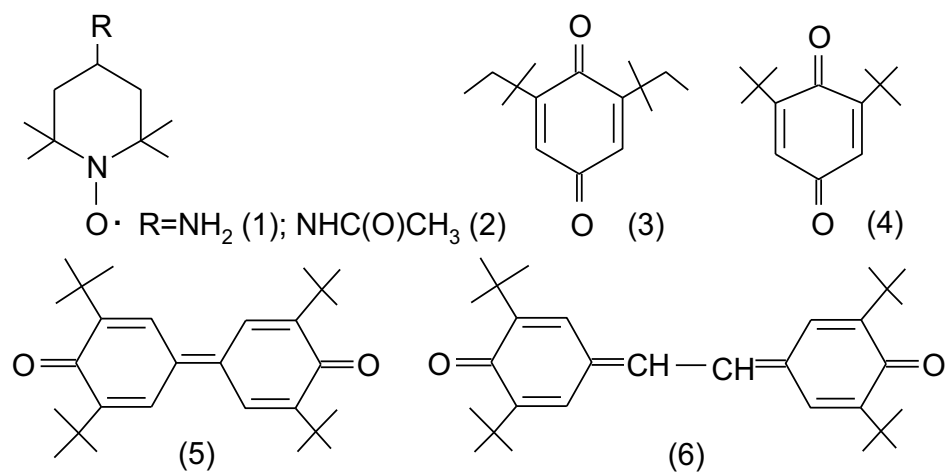

Fig. 3. Scheme.

\section{Discussion of the results}

The main reason for the destruction of PM is the oxidation of the material by air oxygen, with an increase in the rate of this process at elevated temperatures $[3,10]$. The main option to protect the PM from thermo-oxidative destruction is to add a special antioxidant supplement that inhibits the destruction process [4]. A number of scientific papers have been devoted to the study of the mechanism of the stabilising effect of antioxidant supplements and the evaluation of their effectiveness $[4,6,15]$. The most available and actively used are antioxidants from a number of PPF, PZA (HALS), etc. [13, 16]. The oxidation products of these compounds, such as quinones and HP, can also act as inhibitors of oxidative degradation due to their high activity with respect to radical structures. The activity of these substances as PM stabilisers may vary. This is due to the modification of the basic structures in order to improve compatibility with PM, enhance the protectiveinhibitory effect, increase resistance, prolong the duration of action, etc. These circumstances are the way to find new stabilisers and their effective compositions.

The influence of external factors triggers oxidative processes in the PM, leading to destructive changes, reactions of macromolecule chain cross linking, etc. When PM is oxidized, first of all, the accumulation of peroxide structures occurs in it with autoacceleration. It is these active substances that cause destructive changes, which entail changes in the molecular mass distribution and, as a result, a decrease in the mass of the polymer molecule $[10,12]$. The rate of destructive processes is growing uncontrollably and remains unknown. The introduction of a peroxide initiator in PM will allow to simulate the oxidative degradation of PM and to conduct studies of this process at a fixed rate, as well as its kinetic patterns, to determine the length of the polymer chain [10].

It is known that the oxidation of PM on the example of PE in the presence of the initiator benzoyl peroxide or AIBN after an hour (under normal conditions) leads to a 3fold decrease in the molecular weight of the polymer (from 35,000 to 11,000) [17]. Under normal conditions, the rates of oxidative degradation of different PM are very different, and with increasing temperature, their values converge (i.e., level off). The destruction of solid PM (for example, PE, PP, SKI), inhibited by PPF, occurs in accordance with the laws of kinetics characteristic of the destructive oxidation of polymers in solution $[9,10]$.

For a preliminary assessment of the effectiveness of InH for the protection of solid PM, it is possible to study the regularities of the process of inhibiting the oxidative degradation of $\mathrm{PE}$ in solution. A clear indicator of the destruction of polymer chains in solution is the drop in the value of the characteristic viscosity of the PM solution during oxidation [17]. Based on these data, it can be concluded that it is convenient to use easily oxidizing PE for 
research and with its help to study the protective effect of a mixture of stabilisers against oxidative degradation in solution.

Destructive processes in PM begin immediately from the moment of manufacture, and not after a certain period of time associated with the formation, accumulation and subsequent decomposition of hydroperoxide fragments [10]. The presence of an inhibitor in PM inhibits destruction, resulting in an induction period. The duration of the latter determines the effectiveness of the stabiliser $(\mathrm{InH})$.

The influence of InH from a series of HP $(1,2)$ and quinones (3-6) in the presence of the initiator AIBN on destructive changes in PE in order to determine the effectiveness of the action of individual $\mathrm{InH}$ and their synergistic effect was investigated.

In preliminary tests, each of the studied InH showed high protective activity. However, the inhibitory activity of the equimolecular mixed composition $\mathrm{HP}+$ quinone exceeds any single stabiliser, and in this case, the rate of initiated thermo-oxidative destruction of PE is significantly reduced.

The presence of destructive changes in PE was determined by a decrease in the molecular weight of the polymer. The mass change was monitored at regular intervals by measuring the viscosity of the polymer solution. The data of experimental studies of changes in the characteristic viscosity of PE solutions in xylene with an AIBN initiator as a function of time, using 4-AcNH-TEMPO (2) and 2,6-di-tert-amyl-1,4-cyclohexadienone (3) as stabilisers, is shown in Figure 4.

It turned out that the equimolecular mixture of HP (2) and quinone (3) significantly slows down the destructive oxidative processes in PE. The appearance of an induction period and a slight decrease in the viscosity of the PE solution over time (Fig. 4) indicate a quantitative decrease in the destruction of the chains of macromolecules.

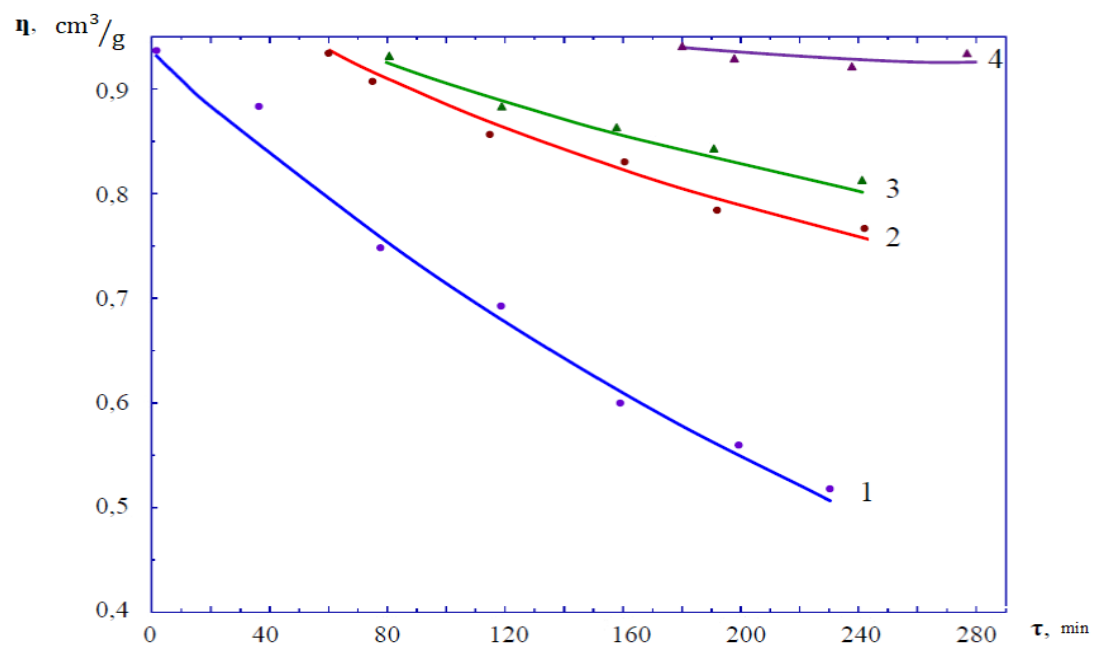

Fig. 4. Kinetics of thermo-destructive oxidation of $P E$ in the presence of an initiator AIBN $\left(\mathrm{C}_{\mathrm{AIBN}}=2 \cdot 10^{-3} \mathrm{~mol} / \mathrm{l}\right)$ and a stabiliser $\left(\mathrm{C}_{\text {stabil. }}=3 \cdot 10^{-3} \mathrm{~mol} / \mathrm{l}\right)$ at $413 \mathrm{~K}: 1-$ no stabiliser; $2-2,6$-di-tertamyl-1,4-cyclohexadienone (3); 3 - 4-acetylamino-2,2,6,6-tetramethylhexahydropyridine-1-oxyl (2); $4-$ a mixture of stabilisers (2) and (3) at a molar ratio of $1: 1$.

The synergism of the action of the mixture of stabilisers (2) and (3) is characterized by a significant increase in the induction $(\tau)$ period (Fig. 4 , curve 4 ), which increases from 0 to 180 minutes, while the ratio must be fulfilled: 


$$
\tau_{\text {general }}>\tau_{1}+\tau_{2}
$$

where $\tau_{1}$ and $\tau_{2}$ are the induction periods of the individual stabilisers HP (3) and quinone (2);

$\tau_{2}$ is the induction period of a mixture of HP (2) and quinone (3).

According to the data obtained (Fig. 1), for the stabiliser HP (2) $\tau_{2}=80 \mathrm{~min}$.; for the stabiliser (3) $\tau_{1}=60 \mathrm{~min}$; $\tau_{2}=180 \mathrm{~min}$. The value of the induction period for the oxidative degradation of PE in the presence of a mixture of stabilisers (2) and (3) exceeds by more than 2 times the induction period in the presence of a more effective stabiliser HP (2), which is proof of the synergistic effect.

The appearance of the induction period is explained by the action of stabilisers from the moment of their introduction into the PM. They are active and capture alkyl and other radical particles. Quinones can be converted into mono-and diesters of hydroquinone [10]. $\mathrm{HP}$, interacting with $\mathrm{PE}$, is reduced to hydroxylamine, which, in turn, is a reducing agent in relation to radicals [10].

The mechanism of synergism of stabilisers is determined by their interaction with each other, with the oxidation products of PM and with the polymer itself. The mechanism of the expected synergistic action of $\mathrm{InH}(2)$ and (3) is shown in Figure 1:

a) breakage of radical chains; inhibiting the action of HP (2):

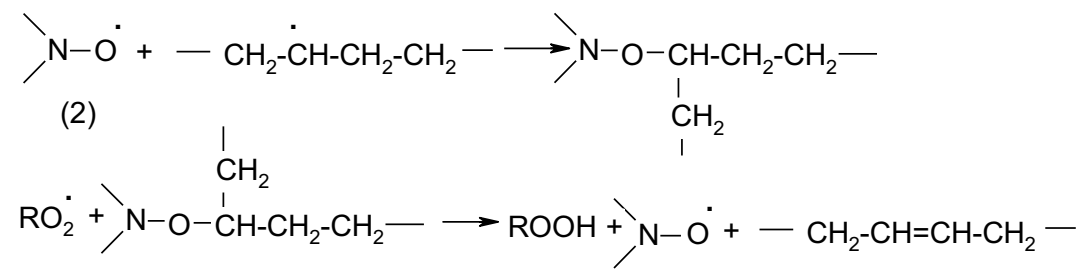

Fig. 5. Scheme.

b) destruction of hydro-peroxide compounds without the formation of radicals; inhibiting the action of quinone (3):<smiles>[R]Oc1cc(CC)c([O])c(C(C)(CC)CCCCCCC)c1</smiles>

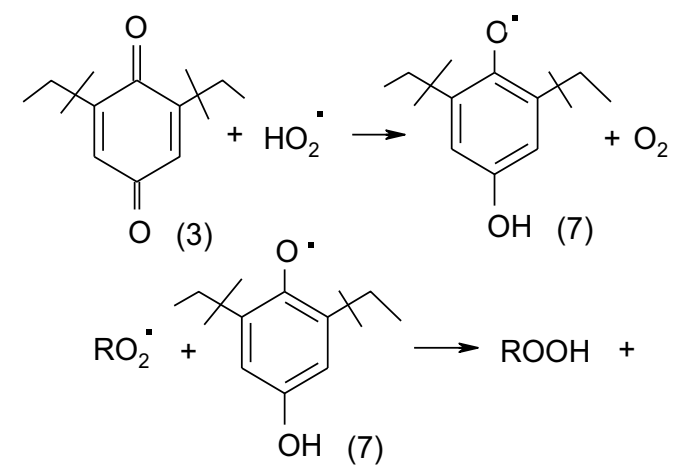

Fig. 6. Scheme. 
where $\mathrm{R} \bullet$ - alkyl radicals; $\mathrm{RO}_{2} \bullet$ - peroxide radicals; $\mathrm{RO} \bullet$ - alkoxyl radicals.

c) regeneration of InH-HP (2) by interaction of radical (7) with hydroxylamine:

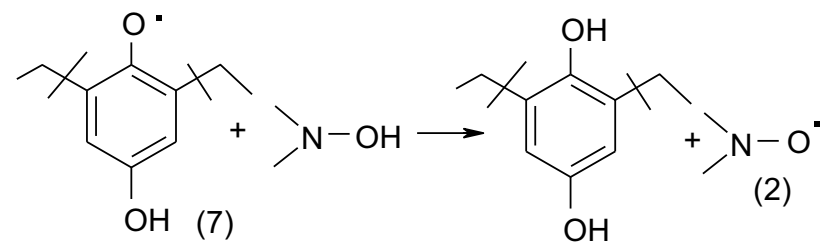

Fig. 7. The proposed mechanism of the stabilising effect of inhibitors (2) and (3) on the thermooxidative destruction of PE.

According to the mechanism, various radical particles of the type $\mathrm{R} \bullet ; \mathrm{RO}_{2} \bullet$; $\mathrm{RO}$ formed as a result of the destruction of PM enter into chemical interaction with the InH of the mixed composition HP (2) and quinone (3) and thereby stabilise the PE. Quinone destroys hydro-peroxide compounds without the formation of radicals (Scheme 1, path b), and HP breaks off radical chains and at the same time regenerates itself (scheme 1, path a, b).

\section{Conclusion}

The synergism of the action of an equimolecular mixture of stabilisers based on quinone and HP against the thermo-oxidative degradation of polyethylene was studied. It is shown that the synergistic effect is to increase the induction period of the mixture of inhibitors by more than 2 times compared to the induction period in the presence of a more effective stabiliser. The mechanism of the joint action of inhibitors in the process of destruction hydro-peroxide connections and breaking the radical chains has been proposed, as well as the mechanism of regeneration of stabilisers.

Thus, the preliminary study showed that the studied mixed compositions of stabilisers effectively slow down the destruction process and can be recommended for use as inhibitors of the thermo-oxidative destruction of polymer materials.

\section{References}

1. E. Yousif, A. Hasan, Journal of taibah university for science 9, 421-448 (2015) doi.org/10.1016/j.jtusci.2014.09

2. G. Wypych, Handbook of material weathering (sixth edition) (2018) doi.org/10.1016/B978-1-927885-31-4.50018-2

3. A. Abdelhafidi, I.M. Babaghayou, S.F. Chabira, M. Sebaa, Procedia - Social and Behavioral Sciences 195, 2922 - 2929 (2015) doi: 10.1016/j.sbspro.2015.09.002

4. V. Ambrogi, C. Carfagna, P. Cerruti, V. Marturano, Additives in Polymers. Modification of Polymer Properties, 87-108 (2017) doi.org/10.1016/B978-0-32344353-1.00004-X

5. J.E. Pickett, Service life prediction of polymers and coatings enhanced methods, 1-18 (2020) doi.org/10.1016/B978-0-12-818367-0.00001-1

6. C. Kröhnke, Polymer science: a comprehensive reference 8, 349375 (2012) doi:10.1016/b978-0-444-53349-4.00212-0

7. R. Pfaendner, Polymer green flame retardants, 419-439 (2014) doi:10.1016/b978-0444-53808-6.00013-5 
8. S. Beißmann, M. Reisinger, K. Grabmayer et al., Polymer degradation and stability 110, 498-508 (2014) doi.org/10.1016/j.polymdegradstab.2014.09.020

9. G. Wypych, Degradation and Stabilisation, 287-412 (2015) doi:10.1016/b978-1895198-85-0.50013-3

10. G. Wypych, PVC Degradation and Stabilisation, 297-430 (2020) doi.org/10.1016/B978-1-927885-61-1.50014-1

11. S. Al-Malaika, F. Axtell, R. Rothon, M. Gilbert, Brydson's Plastics Materials, 127168 (2017) doi:10.1016/b978-0-323-35824-8.00007-4

12. G. Wypych, Handbook of UV Degradation and Stabilisation (Third Edition), 37-71 (2020) doi.org/10.1016/B978-1-927885-57-4.50006-1

13. R.M. Santos, A. Pimenta, G. Botelho, A.V. Machado, Polymer Testing 32(1), 78-85 (2013) doi.org/10.1016/j.polymertesting.2012.08.013

14. M.A. Mercadante, C.B. Kelly, J.M. Bobbitt, L.J. Tilley, N.E. Leadbeater, Nature Protocols (2013) doi.org/10.1038/nprot2013.028

15. L.W. McKeen, The Effect of UV Light and Weather on Plastics and Elastomers (Fourth Edition) Plastics Design Library 2019, 21-47 (2019) doi.org/10.1016/B978-012-816457-0.00002-2

16. P. Gijsman, Polymer Degradation and Stability 145, 2-10 (2017) doi.org/10.1016/j.polymdegradstab.2017.05.012

17. E.S. Petukhova, A.L. Fedorov, Procedia Structural Integrity 20, 75-80 (2019) doi.org/10.1016/j.prostr.2019.12.118 